\title{
Pulmonary Nocardiosis; Similarity to Tuberculosis (A Bacteriological and Proteomics Study)
}

\section{Mogahid M. EI Hassan'; Nageeb S. Saeed²; Mohamed E. Hamid ${ }^{3}$ and M. Goodfellow ${ }^{4}$}

1- College of Medical Laboratory Sciences, Sudan University of Science and Technology, Khartoum, Sudan. E. mail: mogahidelhassan@yahoo.com.

2- Department of Laboratories and Medical Researches, Federal Ministry of Health, Khartoum, Sudan.

3- Department of Microbiology, College of Medicine, King Khalid University, Abha, , Kingdom of Saudi Arabia. P O. Box 641

4- School of Biology, Newcastle University, Newcastle, UK.

\begin{abstract}
Objective: The aims of the present study were to decide the occurrence of nocardia spp. among Sudanese patients suspected with tuberculosis and to investigate all proteins expressed by the genome of Nocardia africana (formerly isolated from patients with pulmonary infection misdiagnosed as MDR and their structures and functions compared to Mycobacterium tuberculosis.

Materials and Methods: Three hundred and twenty-nine patients, presented with pulmonary infection were included in this study. Those patients were examined for the presence of acid- fast bacilli. Two tubes of Lownstein- Jensen (L.J) medium were inoculated with $20 \mu \mathrm{l}$ of the neutralized sputum sample. All cultures were incubated at $37^{\circ} \mathrm{C}$ for 8 weeks before being discarded. Phenotypic characterizations were performed. For nocardia proteom poly acrylamide gele lectrophoresis (PAGE)-based analyses of the four nocardia strains $N$. farcinica SD1828, $N$. africana SD 925, and N. asteroides $\mathrm{N} 317$ are discussed. In-gel tryptic digestion of these isolates was also performed, then the resulting peptides were introduced to MALDI-TOF peptide mass fingerprints were searched using MASCOT software.

Results: Ten isolates showed rapid growth pattern within 2-3 days after inoculation, further conventional methods suggested that all these isolates were belonging to the family nocardiacea. Two Dimentional Poly Acrylamide Gel Electrophoresis (2DPAGE) using pH strips 3-10 revealed that the soluble proteins were visible in a much smaller pI range. All strains exhibited similar protein distributions. A similarity analysis revealed that mycobacterium sequences are of high relevance for the investigated strains.

Conclusions: Nocardia revealed considerable occurrence among patients with pulmonary infections $(3.3 \%)$ giving clinical symptoms similar to those occur by $\mathrm{M}$. tuberculosis infection, this may be due to similarities in functional proteins expressed by their genomes. This finding suggested that pulmonary nocardiosis might occur in patients who suffer from chronic lung disease in Sudan. It is important, therefore, that clinicians in Chest Units should consider this condition, especially when patients with respiratory infections fail to respond to antitubercular therapy.

Nocardiae are Gram-positive aerobic actinomycetes, which are predominantly saprophytic (Orchard, 1981) but also include species forming parasitic association with animals and plants (Goodfellow, 1992). They are in the same family as clinically and industrially important genera such as Mycobacterium, Streptomyces,
\end{abstract}


Corynebacterium and Rhodococcus and they are known to cause a variety of infections in humans and animals. Nocardiae cause a variety of suppurative infections of humans and animals (Ishikawa, 2004; Mogahid et al., 2007). Human infections may be distinguished clinically into cutaneous, subcutaneous, and lymphocutaneous nocardiosis; extrapulmonary nocardiosis; pulmonary nocardiosis; and systemic nocardiosis involving two or more body sites. The incidence of such infections is not known, although nocardiosis has been reported in most regions of the world. Nocardial infections of the internal organs in nontropical countries are mainly caused by Nocardia asteroides, $N$. farcinica, and N. nova; relatively few are caused by $N$. brasiliensis, $N$. otitidiscaviarum, N. pseudobrasiliensis, and N. transvalensis. There have been isolated reports of pulmonary nocardiosis from tropical countries caused by $N$. asteroides, $N$. brasiliensis, $N$. farcinica, $N$. otitidiscaviarum, and $N$. transvalensis, (Koltzscher et al., 2003; Bauer et al., 1966; Gang et al., 2005). According to information from the Center of Disease Control and prevention $80 \%$ of cases present as invasive pulmonary infection, disseminated disease, or brain abscess, and $20 \%$ present as cellulitis. Nocardia asteroides causes at least $50 \%$ of invasive infections. In the United States, estimated 500-1,000 new cases of nocardia infection occur annually. The number of cases has increased with the overall rise in the number of severely immunocompromised persons. Diagnosis is a major challenge. $N$. farcinica frequently is resistant to antimicrobial agents, including the drug of choice trimethoprim-sulfamethoxazole, and has been demonstrated to be more virulent in an animal model. A new combination drug therapy (sulfonamide, ceftriaxone, and amikacin) has shown promise for infections difficult to treat. The hope is that the application of newer molecular diagnostic and subtyping methods may assist in earlier diagnosis and outbreak investigation (www.cd.gov).

Recently, proteomics have been introduced as a new field of research for ultra study of protein structure and function Proteomics is the study of all proteins expressed by a genome. It involves the identification of proteins in the body and the determination of their role in physiological and pathophysiological functions. By proteomics, many new proteins have been identified in different microorganisms and their functions have been well understood (Cutler et al,. 1999, Gorg et al., 2000).

Two-dimensional (2-D) gel electrophoresis of pulse-labeled proteins revealed a global analysis of protein synthesis and turnover in Escherichia coli (Weichart et al., 2003). Comparative proteomics of the human pathogen Campylobacter jejuni revealed an important first step in characterizing strain differences potentially responsible for different disease outcomes associated with this organism (Brubacher et al., 2003). Several studies subjected Mycobacterium tuberculosis to comprehensive proteomic analysis. Culture supernatant proteins of virulent Mycobacterium tuberculosis $\mathrm{H}_{37} \mathrm{Rv}$ and attenuated Mycobacterium bovis BCG were comprehensively analyzed using proteomics techniques (Mattow et al., 2001). In another study, proteomics revealed open reading frames in Mycobacterium tuberculosis $\mathrm{H}_{37} \mathrm{Rv}$ not predicted by genomics (Peter et al., 2003). This clearly showed that, analysis of proteins directly may come up with new knowledge which may completely alter the approaches to disease diagnosis, control and treatment. Definitive diagnosis of nocardiosis depends on the isolation and identification of the causative organism from clinical materials. These procedures are not straightforward; hence the true incidence of the disease is masked, a problem which is compounded by poor documentation. Generally, nocardia cases are difficult to diagnose (Garcia-Benitez et al., 2001). 


\section{MATERIALS AND METHODS Collection of the Samples}

Three hundred and twenty- nine patients, who were attending Abu-Anga Teaching Hospital, El-Shaab Teaching Hospital and the National Health Laboratory, in the Sudan during the period from October 2004 to January 2006, were examined for the presence of acid-fast bacilli. They were suspected of having tuberculosis infection according to the symptoms. Most of the patients had either not responded to treatment with antitubercular drugs or had responded and then relapsed. Sputum samples were collected according to WHO criteria in sterile, plastic widemouthed, strong leak-proof containers. Following treatment with the digestiondecontamination procedure of Roberts et al., 1991, the sputum samples were concentrated by centrifugation and the resultant preparations were used to inoculate Lowenstein-Jensen (LJ) slopes, which were incubated at $37^{\circ} \mathrm{C}$ for 14 days and then used to make smears, which were examined with a standard Ziehl-Neelsen acid-fast stain.

\section{Phenotypic Characterization}

Ten of the LJ slopes supported the growth of small orange filamentous colonies, which were considered to be typical of nocardiae. The isolates, which were designated SD1001, SD1002, SD1002, SD1003, SD1004, SD1005, SD1006, SD1007, SD1008, SD1009 and SD1010, were subcultured and maintained on glucose-yeast extract agar (GYEA) slopes at room temprature. The ten isolates were examined for a range of phenotypic properties described by Isik et al., 1999. Standard procedures were also used for the extraction and analysis of mycolic acids as described by Minnikin, et al., 1975 and Nocardia africana strains used as controls.

\section{Preparation on Nocardia Lysate}

The test strains were cultivated on GYEA medium (Appendex I1) for 2-3 days at $37^{\circ} \mathrm{C}$ then checked for purity. Nocardia species were then harvested, inactivated in the water path at $90 \mathrm{oC}$ for one hour and the cells were washed with sterile phosphate buffer saline (P.B.S) and then centrifuged at $15000 \mathrm{rpm}$ for 5 minutes. Supernatants were discarded and the pellet was washed by resuspending in MilliQ water and centrifuged again at $10000 \mathrm{rpm}$ for 10 minutes, and the supernatant was discarded.

Then, $1 \mathrm{ml}$ of urea lysis buffer 8 M (Appendix IV) was added to each sample in eppendorf tubes in addition to $100 \mu \mathrm{l}$ of protease inhibitor (CALBIOCHEM, Cat No 539134), $20 \mu \mathrm{l}$ of (500 U) benzonase (Novagen Lot $\mathrm{N}$ $62211-1)$ and $0.5 \mathrm{gm}$ of silica beads (0.1mm, Cat No 11079101 Z- Biospec products, Inc). The mixture was then vortexed for one minute (Vortex Genie 2 Model G.560E Bohemia USA).

Samples were then lysed by freezing in liquid nitrogen and thawing in $37 \mathrm{oC}$ in waterpath, this step was repeated for 5 times. Samples were then put on a shaker (Type MM 300 cat No 85720 $\mathrm{GmbH}$ and co KG, Germany) with a frequency of 3000 per minute for 30 minutes. Step of freeze-thawing was repeated again for other 5 times and the shaking step was also repeated as previously described. Samples were then spin down at $15000 \mathrm{rpm}$ for 5 minutes, supernatant (which contain the soluble proteins) was collected and protein concentration was measured with spectrophotometer (Model 8024-0600, From Pharmacia Biotech, England).

Two Dimensional Gel Electrophoresis

Seventy (70) $\mu \mathrm{g}$ of protein were Loaded on immobilized $\mathrm{pH}$ gradients (I PG)

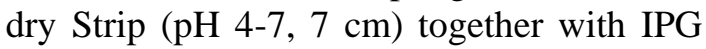
buffer (pH 3- 10) and $20 \mathrm{mM}$ DTT), overnight passive rehydration was performed for the dry strip then Ettan I PG phor (Amersham Bioscience) was used to a achieve First dimension Iso Electrical Focusing. A second dimension SDS - PAGE 
was done by using Hofer miniv E8 $\times 9 \mathrm{~cm}$ gels device accrding to Laemmli.1970

\section{Tryptic Digestion and Mass Spectrometric Sequencing}

In-solution tryptic digestion of nocardia isolates lysate was also performed; a total of $4 \mu$ l containing approximately 250 $\mu \mathrm{g}$ ) of the lysate was dry with vacuum centrifuge, the dry protein was redisolved in $250 \mu \mathrm{l}$ of $8 \mathrm{M}$ urea, $0.4 \mathrm{M} \mathrm{N}_{4} \mathrm{HCO}_{3}$ followed by $25 \mu \mathrm{l}$ of $45 \mathrm{mM}$ dithiothereitol the mixture was incubated at $50{ }^{0} \mathrm{C}$ fore 15 minutes, 25 $\mu \mathrm{L}$ of $100 \mathrm{mM}$ Iodoactamide were added and the mixture was incubated at ambient temperature in darkness for additional 15 minutes, $700 \mu \mathrm{L}$ of deionized water was added together with 5\% (w/w) trypsin (12.5 $\mu \mathrm{g})$. The digestion was performed at $37^{\circ} \mathrm{C}$ overnight in darkness. Resulting peptides were then desalted by using Zip Tip column (microbed $\mathrm{C}_{18}$; Millipore, USA), then the peptides were introduced to mass spectrophotometer (QSTAR from Applied Biosystem), peptide mass fingerprints were searched using MASCOT software.

\section{RESULTS \\ Bacteriology Results}

All positive smears showed positive growth on LJ medium after 2-21 days post inoculation. The colonial morphology of 319 (97\%) appeared as rough, friable, warty, granular and grey in color with irregular margins and showed the appearance of AFB when stained again (indirect smear) with $\mathrm{ZN}$ staining procedure for more confirmation. The 319 isolates were initially identified as members of the Mycobacterium tuberculosis complex.

Ten $(3 \%)$ of the LJ slopes reveled the growth of small orange filamentous colonies, which were tentatively considered to be nocardiae (Fig. 1).

The 10 nocardia isolates, which were then designated SD1001, SD1002, SD1003, SD1004, SD1005, SD1006, SD1007, SD1008, SD1009 and SD10010 were subcultured and maintained on glucose-yeast extract agar (GYEA) slopes at room temperature and as suspensions of mycelial fragments in glycerol $(20 \%$ [vol/ vol] $)$ at $-70^{\circ} \mathrm{C}$. The 10 isolates were studied phenotypically.
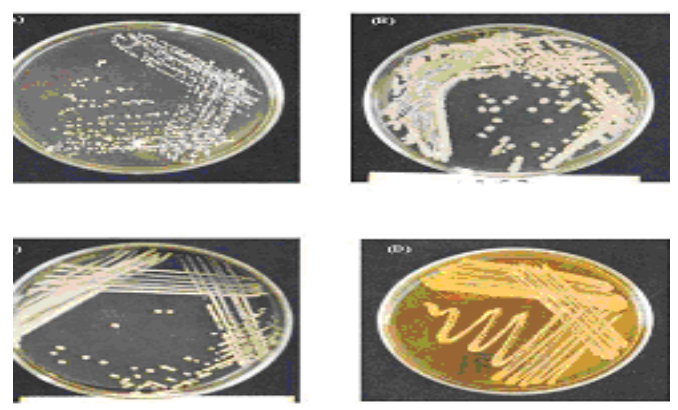

Fig. 1: Growth of N. africana SD 1002 (A); SD 1003 (B); SD 925 (C); N. farcinica SD 1819 (D); on glucose yeast extract agar medium.7 days old cultures.

\section{Biochemical tests for nocardia isolates}

Selected biochemical tests were performed. The result for these tests showed that all the strains utilize glucose by oxidation pathway and that they were all catalase positive and also positive for urea. Concerning growth at $45^{\circ} \mathrm{C}, 7$ out of the ten were positive $(70 \%)$. Only $2(20 \%)$ were positive for manitol and rhaminose as well as starch whilst all the isolates were negative for xanthine, casein, tyrosine, sorbitol, arabinose and citrate. Regarding mycolic acids, all tested isolates showed the standard patterns of mycolic acid components using thin layer chromatographic technique.The tested strains were found to have phenotypic properties typical of members of the genus nocardia ( Figs 1 and 2).

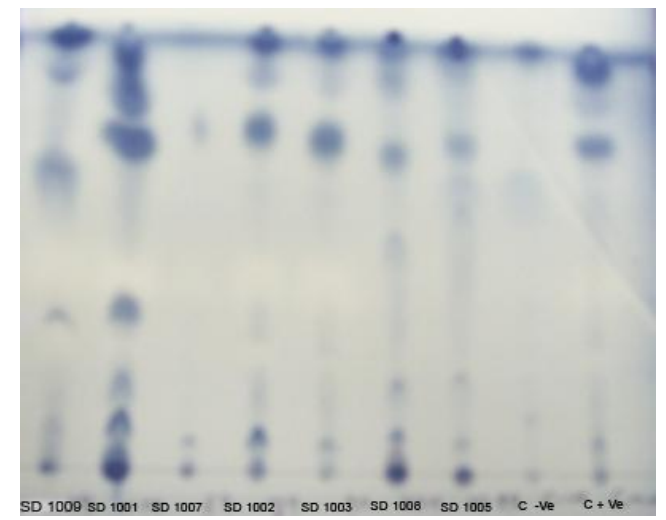

Fig. 2: Thin layer chromatography (TLC) $(10 \times 10 \mathrm{~cm})$, run twice in a solvent containing ToluenAceton (97:3, V/V), C +Ve: N. africana SD 925, C-Ve: S. aurues. 


\section{Proteomic Results}

The results of 2-D gel electrophoresis revealed a total of hundreds of protein spots for nocardia lysate. The molecular weights range between $25-75 \mathrm{kDa}$ and the Iso-electric point (pI) of these range between 4-7 (Figs 3 and 4).

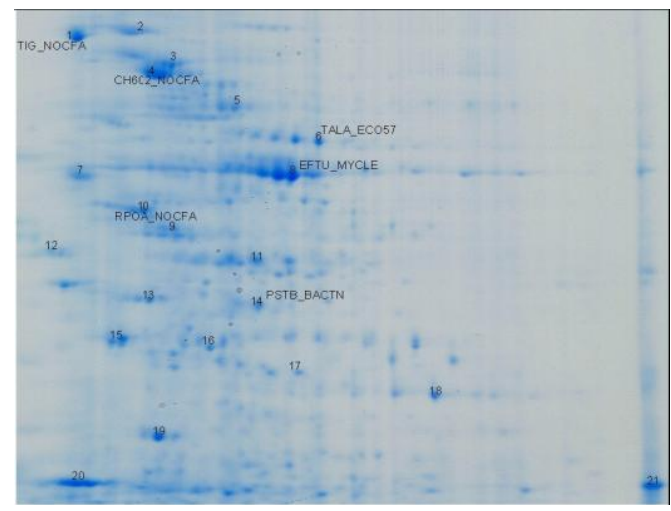

Fig. 3: Representative spot assignments for $N$. farcinica SD1828, pH 4-7, MW 25-75 kDa.

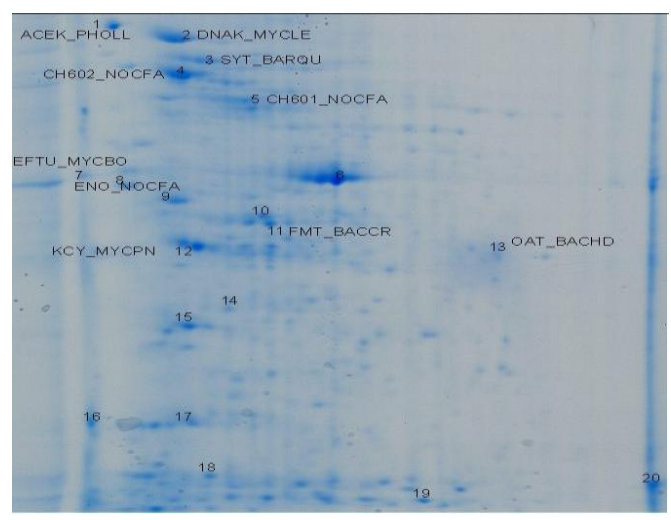

Fig. 4: Representative spot assignments for $N$. africana. SD 925, pH 4-7, MW 25-75 kDa.
On the other hand, different peptides were detected by mass spectrometer. Alignment of those peptides was performed on Swiss-Prot. The resulting data revealed highly significant homology with Mycobacterium tuberculosis (set of Table 1).

\section{CONCULOSION}

A relatively large sample size (329) was analyzed in this study compared with previous studies in Sudan conducted in the same field and nocardia species has constituted $3 \%$ of all isolate. A significant resistant pattern $(40 \%)$ were observed through these ten (10) isolates, hence more attention should be drawn towards patients who did not respond to anti-tuberculosis therapy, as other pathogens, including Nocardia spp. may be the cause of the infection.

Hundreds of nocardia protein spots were captured on stained gel. Some proteins were identified in a gel digest using QSTAR instrument analysis of the results showed significant similarities between nocardia and Mycobacterium tuberculosis; this may provide evidences for best understanding of the pathogenesis and increase the knowledge with respect to disease treatment and vaccination. 
Table 1/1: Elongation factor Tu of Mycobacterium leprae EFTU_MYCLE (P30768)

\begin{tabular}{|c|c|c|}
\hline Description & SwissProt Accession \# & Organism \\
\hline$\_$MYCPA & Q73SD1 & Mycobacterium paratuberculosis \\
\hline EFTU_MYCTU & P0A558 & Mycobacterium tuberculosis \\
\hline EFTU_MYCBO & P0A559 & Mycobacterium bovis \\
\hline _MYCSS & Q1BDD3 & Mycobacterium sp. (strain MCS) \\
\hline$\_9 \mathrm{MYCO}$ & Q1T9Z3 & Mycobacterium sp. KMS \\
\hline$\_9 \mathrm{MYCO}$ & Q1TV69 & Mycobacterium sp. JLS \\
\hline _MYCVN & Q262Y2 & Mycobacterium vanbaalenii PYR-1 \\
\hline$\_$MYCFV & Q27DQ3 & Mycobacterium flavescence \\
\hline _NOCFA & Q5YPG4 & Nocardia farcinica \\
\hline
\end{tabular}

Sequence comparison EFTU_MYCLE and Q5YPG4_NOCFA

Query: 1 MAKAKFERTKPHVNIGTIGHVDHGKTTLTAAITKVLHDKFPNLNESRAFDQIDNAPEERQ 60 +AKAKFERTKPHVNIGTIGHVDHGKTTLTAAITKVL DK+P+LN+S AFDQID APEE+

Sbjct: 20 VAKAKFERTKPHVNIGTIGHVDHGKTTLTAAITKVLADKYPDLNQSFAFDQIDKAPEEKA 79

Query: 61 RGITINISHVEYQTEKRHYAHVDAPGHADYIKNMITGAAQMDGAILVVAATDGPMPQTRE 120 RGITINISHVEYQTEKRHYAHVDAPGHADYIKNMITGAAQMDGAILVVAATDGPMPQTRE

Sbjct: 80 RGITINISHVEYQTEKRHYAHVDAPGHADYIKNMITGAAQMDGAILVVAATDGPMPQTRE 139

Query: 121 HVLLARQVGVPYILVALNKSXXXXXXXXXXXXXXXXXXXXXXQEFDEDAPVVRVSALKAL 180 HVLLARQVGVPYILVALNK+ QEFDE+APVVRVS LKAL

Sbjct: 140 HVLLARQVGVPYILVALNKADMVDDEEILELVEMEVRELLAAQEFDEEAPVVRVSGLKAL 199

Query: 181 EGDAKWVESVTQLMDAVDESIPAPVRETDKPFLMPVEDVFXXXXXXXXXXXXXXXXXXXX 240 EGD KWV+SV LMDAVDESIP PVRETDKPFLMP+EDVF

Sbjct: 200 EGDPKWVKSVEDLMDAVDESIPDPVRETDKPFLMPIEDVFTITGRGTVVTGRVERGIINV 259

Query: 241 XXXXXXXXXXQXXXXXXXXGVEMFRKLLDQGQAGDNVGLLLRGIKREDVERGQVVIKPGT 300 G+EMFRKLLDQGQAGDNVGLL+RGIKREDVERGQVVIKPGT

Sbjct: 260 NEEVEITGIRPETTKTTVTGIEMFRKLLDQGQAGDNVGLLIRGIKREDVERGQVVIKPGT 319

Query: 301 TTPHTEFEGQVYILSKDEGGRHTPFFNNYRPQFYFRTTDVTGVVTLPEGTEMVMPGDNTN 360 TTPHTEFEGQ YILSKDEGGRHTPFFNNYRPQFYFRTTDVTGVVTLPEGTEMVMPGDNT

Sbjct: 320 TTPHTEFEGQAYILSKDEGGRHTPFFNNYRPQFYFRTTDVTGVVTLPEGTEMVMPGDNTE 379

Query: 361 ISVTLIQPVAMDEGLRFAIREGGRTVGAGRVVKIIK 396 +SV LIQPVAM+EGLRFAIREGGRTVGAGRV KIIK

Sbjct: 380 MSVKLIQPVAMEEGLRFAIREGGRTVGAGRVTKIIK 41 
Table 1/2: Chaperone protein dnaK, HSP70 of Mycobacterium paratuberculosis DNAK_MYCPA (Q00488)

\begin{tabular}{|l|l|l|}
\hline \multicolumn{1}{|r|}{ Description } & SwissProt Accession \# & Organism \\
\hline DNAK_MYCLE & P19993 & Mycobacterium leprae \\
\hline DNAK_MYCTU & P0A5B9 & Mycobacterium tuberculosis \\
\hline DNAK_MYCBO & P0A5C0 & Mycobacterium bovis \\
\hline MYCVN & Q25TL7 & Mycobacterium vanbaalenii PYR-1 \\
\hline _MYCFV & Q27CE8 & Mycobacterium flavescence PYR-GCK \\
\hline _MYCSS & Q1BEV1 & Mycobacterium sp. strain MCS \\
\hline -9MYCO & Q1TWF2 & Mycobacterium sp. JLS \\
\hline -9MYCO & Q1TDC6 & Mycobacterium $s p$. KMS \\
\hline DNAK_NOCFA & Q5YNI0 & Nocardia farcinica \\
\hline
\end{tabular}

Sequence comparison DNAL_MYCPA and DNAK_NOCFA

Query: 1 ARAVGIDLGTTNSVVAVLEGGDPVVVANSEGSRTTPSIVAFARNGEVLVGQPAKNQAVTN 60 ARAVGIDLGTTNSVVAVLEGG+PVVVANSEGSRTTPSIVAFA+NGEVLVGQPAKNQAVTN

Sbjct: 2 ARAVGIDLGTTNSVVAVLEGGEPVVVANSEGSRTTPSIVAFAKNGEVLVGQPAKNQAVTN 61

Query: 61 VDRTIRSVKRHMGTDWSIEIDGKKYTAQEISARVLMKLKRDAEAYLGEDITDAVITVPAY 120 VDRTIRSVKRH+GTDW++EIDGKKYT QEISAR LMKLKRDAEAYLGE+ITDAVITVPAY

Sbjct: 62 VDRTIRSVKRHIGTDWTVEIDGKKYTPQEISARTLMKLKRDAEAYLGEEITDAVITVPAY 121

Query: 121 FNDAQRQATKEAGQIAGLNVLRIVNEPTAAALAYGLDKGEKEQTILVFDLGGGTFDVSLL 180 F DAQRQATKEAGQIAGLNVLRIVNEPTAAALAYGLDKG+KEQTILVFDLGGGTFDVSLL

Sbjct: 122 FEDAQRQATKEAGQIAGLNVLRIVNEPTAAALAYGLDKGDKEQTILVFDLGGGTFDVSLL 181

Query: 181 EIGEGVVEVRATSGDNQLGGDDWDDRIVNWLVDKFKGTSGIDLTKDKMAMQRLREAAEKA 240 EIGEGVVEVRATSGDN LGGDDWD RIVNWLVDKFK +SGIDLTKDKMAMQRLREAAEKA

Sbjct: 182 EIGEGVVEVRATSGDNHLGGDDWDQRIVNWLVDKFKASSGIDLTKDKMAMQRLREAAEKA 241

Query: 241 KIELSSSQSTSINLPYITVDADKNPLFLDEQLTRAEFQRITQDLLDRTRQPFKSVIADAG 300 KIELSSSQSTSINLPYITVDADKNPLFLDEQL+RAEFQ+IT DLLDRTR PF+ VI DAG

Sbjct: 242 KIELSSSQSTSINLPYITVDADKNPLFLDEQLSRAEFQKITSDLLDRTRAPFQQVIKDAG 301

Query: 301 ISVSDIDHVVLVGGSTRMPAVTDLVKELTGGKEPNKGVNPDEXXXXXXXXXXXXXKGEVK 360 ISVSDIDHVVLVGGSTRMPAV+DLV+ELTGGKEPNKGVNPDEKGEVK

Sbjct: 302 ISVSDIDHVVLVGGSTRMPAVSDLVRELTGGKEPNKGVNPDEVVAVGAALQAGVLKGEVK 361

Query: 361 DVLLLDVTPLSLGIETKGGVMTKLIERNTTIPTKRSETFTTADDNQPSVQIQVYQGEREI 420 DVLLLDVTPLSLGIETKGGVMTKLIERNTTIPTKRSETFTTADDNQPSVQIQV+QGEREI

Sbjct: 362 DVLLLDVTPLSLGIETKGGVMTKLIERNTTIPTKRSETFTTADDNQPSVQIQVFQGEREI 421

Query: 421 AAHNKLLGSFELTGIPPAPRGVPQIEVTFDIDANGIVHVTAKDKGTGKENTIKIQEGSGL 480 AAHNKLLGSFELTGIPPAPRGVPQIEVTFDIDANGIVHVTAKDKGTGKENTIKIQ+GSGL

Sbjct: 422 AAHNKLLGSFELTGIPPAPRGVPQIEVTFDIDANGIVHVTAKDKGTGKENTIKIQDGSGL 481

Query: 481 SKEEIDRMIXXXXXXXXXXXXXXXXXXVRNQAESLVYQTEKFVKDQREAEGGSKVPEETL 540 SKEEIDRMIRNQAE+LV+QTEKF+KD+KVP+

Sbjct: 482 SKEEIDRMIKDAEQHAAEDKARREEAETRNQAETLVHQTEKFIKDNED-----KVPADVK 536

Query: 541 SKVDAAIADAKTALGGTDITAIKSAMEKLGQESQALGQAIYEATQAE 587 SKV+AAIA+AALGTDIA+K+A+EKLESQALGQAIYEAA+

Sbjct: 537 SKVEAAIAEANEALAGTDIAAVKAAVEKLATESQALGQAIYEAQGAD 583 
Table 1/3: 60 kDa chaperonin 1 of $N$. farcinica CH601_NOCFA (Q5Z1F9)

\begin{tabular}{|l|l|l|}
\hline Description & SwissProt Accession \# & Organism \\
\hline _RHOSR & Q0S3B9 & Rhodococcus sp. strain RHA1 \\
\hline _MYCSS & Q1BCW7 & Mycobacterium sp. strain MCS \\
\hline 9MYCO & Q1TR57 & Mycobacterium sp. JLS \\
\hline -9MYCO & Q1TCR3 & Mycobacterium sp. KMS \\
\hline _MYCVN & Q263S8 & Mycobacterium vanbaalenii PYR-1 \\
\hline _MYCFV & Q27E56 & Mycobacterium flavescence PYR-GCK \\
\hline CH601_MYCPA & P60545 & Mycobacterium paratuberculosis \\
\hline CH601_MYCTU & P0A518 & Mycobacterium tuberculosis \\
\hline CH601_MYCBO & P0A519 & Mycobacterium bovis \\
\hline CH601_MYCLE & P37578 & Mycobacterium leprae \\
\hline
\end{tabular}

Sequence comparison CH601_NOCFA and Q0S3B9_RHOSR and Q1BCW7_MYCSS

Query: 1 MAKQIEFDEKARRALERGVDKLADAVKVTLGPRGRHVVLAKAFGGPTVTNDGVTIARDID 60 M+KQIEF+E ARR+LERGVDKLADAVKVTLGPRGRHVVLAKAFGGPTVTNDGV+IAR+I+ Sbjct: 1 MSKQIEFNEVARRSLERGVDKLADAVKVTLGPRGRHVVLAKAFGGPTVTNDGVSIAREIE 60 M+KQIEF+E ARRA+E GVDKLADAVKVTLGPRGR+VVLAK++GGPTVTNDGVTIAR+ID

Sbjct: 1 MSKQIEFNETARRAMEIGVDKLADAVKVTLGPRGRNVVLAKSWGGPTVTNDGVTIAREID 60

Query: 61 LEDPFENLGAQLVKSVATKTNDVAGDGXXXXXXXXXXXXRGGLKNVAAGANPIAVGSGIX 120 LEDPFENLGAQLVKSVATKTNDVAGDGRGGLKN+AAGANP+A+G GI

Sbjct: 61 LEDPFENLGAQLVKSVATKTNDVAGDGTTTATVLAQAIVRGGLKNIAAGANPMALGIGIN 120 LEDPFENLGAQLVKSVATKTNDVAGDGR GL+NVAAGANPIA+G+GI

Sbjct: 61 LEDPFENLGAQLVKSVATKTNDVAGDGTTTATVLAQALVRAGLRNVAAGANPIALGAGIS 120

Query: 121 XXXXXXXXXXXXXXTPVSGEQAIAQVATVSSRDEEIGEMVGKALTTVGKDGVVTVEESST 180 PV G+ +IAQVATVSSRDEEIGEMVG+ALT VG DGVVTVEESST

Sbjct: 121 AAADKVVEALLAAAKPVEGKTSIAQVATVSSRDEEIGEMVGEALTRVGTDGVVTVEESST 180 TPV + IAQVATVSSRDE+IGE+VG+A+T VG DGVVTVEESST

Sbjct: 121 KAADAVSEALLAAATPVDDKSGIAQVATVSSRDEQIGELVGEAMTKVGHDGVVTVEESST 180

Query: 181 LQTELVVTEGVQFDKGYLSPYFITDTDTQEAVLEDAFVLLHREKISSLPDLLPLLEKIAE 240 L TELV+TEGVQFDKGYLSPYF+TD D Q+AV EDA VLL+REKI+SLPD LPLLEK+AE

Sbjct: 181 LATELVITEGVQFDKGYLSPYFVTDLDAQKAVYEDALVLLYREKITSLPDFLPLLEKVAE 240 L TEL VTEGV FDKG++S YF+TD D+QEAVLEDA VLLHREK+SSLPDLLPLLEK+AE

Sbjct: 181 LNTELEVTEGVGFDKGFISAYFVTDFDSQEAVLEDALVLLHREKVSSLPDLLPLLEKVAE 240

Query: 241 AGKPVLIVAEDVEGEALSTLVVNSIRKTLKAVAVKAPFFGDRRKAFLDDLAVVTAGTVVN 300 +GKP+LI+AEDVEGE LSTLVVNSIRKT+KAVAVKAPFFGDRRKAFLDDLAVVT GTV+N

Sbjct: 241 SGKPLLIIAEDVEGEVLSTLVVNSIRKTIKAVAVKAPFFGDRRKAFLDDLAVVTGGTVIN 300 AGKP+LI+AEDVEGEALSTLVVN+IRKTLKAVAVKAPFFGDRRKAFLDDLAVVT G V+N

Sbjct: 241 AGKPLLIIAEDVEGEALSTLVVNAIRKTLKAVAVKAPFFGDRRKAFLDDLAVVTGGQVIN 300

Query: 301 PDLGITLREAGIDVLGKARRVVVTKDETTIIDGAGTAEDIAARAAQLRREIEATDSDWDR 360 D+G+TL++AG+D+LG ARRVVV+KDETTI+DGAGT +DI R AQLRREIE TDSDWDR

Sbjct: 301 SDVGLTLKDAGLDLLGSARRVVVSKDETTIVDGAGTDDDIKGRVAQLRREIENTDSDWDR 360 PD+G+ LRE G+DVLG ARRVVVTKD T I+DG G+A+ IA RA QLR EIEATDSDWDR

Sbjct: 301 PDVGLVLREVGLDVLGTARRVVVTKDSTVIVDGGGSADAIADRAKQLRAEIEATDSDWDR 360

Query: 361 EKLEERLXXXXXXXXXXXXXXXTETALKERKYRVEDAVSAAKAAVDEGIVPGGGTALVQA 420 EKLEERLTET LKERK+RVEDAV+AAKAAV EGIVPGGG+ALVQA

Sbjct: 361 EKLEERLAKLAGGVAVIKVGAATETDLKERKFRVEDAVNAAKAAVAEGIVPGGGSALVQA 420 EKLEERLTET LK+RK VEDAVSAAKAAV+EGIV GGG ALVQA

Sbjct: 361 EKLEERLAKLAGGVAVIKVGAATETDLKKRKEAVEDAVSAAKAAVEEGIVTGGGAALVQA 420

Query: 421 ATKLVELRDSLSGDEAVGVEVVRKALEAPLFWIASNAGLDGAVVVSKVAEGKE--GFNAA 478 $+\mathrm{T}+\mathrm{L}+\mathrm{L}+\mathrm{GDEA} \mathrm{GV}+\mathrm{VVR}+\mathrm{AL}+\mathrm{APL}+\mathrm{WIASNAGLDG}+\mathrm{VV}$ SKVAE +GFNAA

Sbjct: 421 STELAD-NLGLTGDEATGVKVVREALQAPLYWIASNAGLDGSVVTSKVAEQPKGHGFNAA 479 L LR S+SGDEA+GVEVAL APL+WIA+NAGLDG+VVV+KV+E +GFNAA

Sbjct: 421 RKALDSLRGSVSGDEALGVEVFNSALSAPLYWIATNAGLDGSVVVNKVSELPAGQGFNAA 480

Query: 479 TLSYGDLLTDGVVDPVKXXXXXXXXXXXXXXMVLTTESAVVDKPAEEAEDHSHHGHAH 536 TL+YGDLL DGVVDPVKM+LTTESAVV+KPAEE E + HGH+H

Sbjct: 480 TLTYGDLLADGVVDPVKVTRSAVVNAASVARMILTTESAVVEKPAEENEQQTGHGHSH 537

TL +GDLL DGVVDPVKMVLTTE+A+VDKPAEE EDH HHGHA

Sbjct: 481 TLEFGDLLADGVVDPVKVTRSAVLNAASVARMVLTTETAIVDKPAEE-EDHGHGHHHGHA 539

Query: 536 H 536

Sbjct: 540540

$\mathrm{H}$

Sbjct: 540 H 540 
Table 1/4: $60 \mathrm{kDa}$ chaperonin 2 of $N$. farcinica CH602_NOCFA (Q9AFA6)

\begin{tabular}{|c|c|c|}
\hline Description & SwissProt Accession \# & Organism \\
\hline CH60_COREQ & Q93Q12 & Corynebacterium equii (Rhodococcus equi) \\
\hline _RHOSR & Q0SET3 & Rhodococus sp. strain RHAI \\
\hline MYCFV & Q27CR6 & Mycobacterium flavescence PYR-GCK \\
\hline MYCSS & Q1BEF6 & Mycobacterium sp. strain MCS \\
\hline 9MYCO & Q1TWU6 & Mycobacterium sp. JLS \\
\hline _MYCVN & Q267S7 & Mycobacterium vanbaalenii PYR-I \\
\hline _9MYCO & Q1TDR8 & Mycobacterium sp. KMS \\
\hline _9MYCO & Q8GAR8 & Mycobacterium sp. 185-409 \\
\hline CH60_NOCAS & Q9AFC5 & Nocardia asteroides \\
\hline _MYCMR & Q8G8X0 & Mycobacterium marinum \\
\hline CH602_MYCPA & $\mathbf{P 4 2 3 8 4}$ & Mycobacterium paratuberculosis \\
\hline
\end{tabular}

Sequence comparison CH602_NOCFA and CH60_NOCAS and CH602_MYCPA

Query: 1MAKTIAYDEEARRGLERGLNSLADAVKVTLGPKGRNVVLEKKWGAPTITNDGVSIAKEIE 60 MAKTIAYDEEARRGLERGLNSLADAVKVTLGPKGRNVVLEKKWGAPTITNDGVSIAKEIE

Sbjct: 1MAKTIAYDEEARRGLERGLNSLADAVKVTLGPKGRNVVLEKKWGAPTITNDGVSIAKEIE 60

Query: 2 AKTIAYDEEARRGLERGLNSLADAVKVTLGPKGRNVVLEKKWGAPTITNDGVSIAKEIEL 61 AKTIAYDEEARRGLERGLN+LADAVKVTLGPKGRNVVLEKKWGAPTITNDGVSIAKEIEL

Sbjct: 1 AKTIAYDEEARRGLERGLNALADAVKVTLGPKGRNVVLEKKWGAPTITNDGVSIAKEIEL 60

Query: 61 LEDPYEKIGAELVKEVAKKXXXXXXXXXXXXXXXXXXXXREGLRNVAAGANPLGLKRGIE 120 LEDPYEKIGAELVKEVAKK REGLRNVAAGANPLG KRGIE

Sbjct: 61 LEDPYEKIGAELVKEVAKKTDDVAGDGTTTATVLAQALVREGLRNVAAGANPLGRKRGIE 120 Query: 62 EDPYEKIGAELVKEVAKKXXXXXXXXXXXXXXXXXXXXREGLRNVAAGANPLGLKRGIEK 121 EDPYEKIGAELVKEVAKKREGLRNVAAGANPLGLKRGIEK

Sbjct: 61 EDPYEKIGAELVKEVAKKTDDVAGDGTTTATVLAQALVREGLRNVAAGANPLGLKRGIEK 120

Query: 121 KAVEAVTAKLLDTAKEVETKEQIAATAGISAGDASIGELIAEAMDKVGKEGVITVEESNT 180 KAVEAVTAKLLDTAKEVETKEQIAATAGISAGDA+IGELIAEAMDKVGKEGVITVEESNT

Sbjct: 121 KAVEAVTAKLLDTAKEVETKEQIAATAGISAGDAAIGELIAEAMDKVGKEGVITVEESNT 180 Query: 122 AVEAVTAKLLDTAKEVETKEQIAATAGISAGDASIGELIAEAMDKVGKEGVITVEESNTF 18 AVE VT LL +AKEVETK+QIAATA ISAGD SIG+LIAEAMDKVG EGVITVEESNTF

Sbjct: 121 AVEKVTETLLKSAKEVETKDQIAATAAISAGDQSIGDLIAEAMDKVGNEGVITVEESNTF 180

Query: 181 FGLQLELTEGMRFDKGYISGYFVTDPERQEAVLEDPYILLVGSKVSTVKDLLPLLEKVIQ 240 FGLQLELTEGMRFDKGYISGYF TDPERQEAVLEDPYILLVGSKVSTVKDLLPLLEKVIQ

Sbjct: 181 FGLQLELTEGMRFDKGYISGYFATDPERQEAVLEDPYILLVGSKVSTVKDLLPLLEKVIQ 240

Query: 182 GLQLELTEGMRFDKGYISGYFVTDPERQEAVLEDPYILLVGSKVSTVKDLLPLLEKVIQA 241 GLQLELTEGMRFDKGYISGYFVTD ERQEAVLEDP+ILLV SKVSTVKDLLPLLEKVIQA

Sbjct: 181 GLQLELTEGMRFDKGYISGYFVTDAERQEAVLEDPFILLVSSKVSTVKDLLPLLEKVIQA 240

Query: 241 AGKPLLIIAEDVEGEALSTLVVNKIRGTFKSVAVKAPGFGDRRKAQLADIAILTGGEVIS 300 AGKPLLIIAEDVEGEALSTLVV KI GTFKSVAVKAPG GDRRKAQLADIAILTGG+VIS

Sbjct: 241 AGKPLLIIAEDVEGEALSTLVVKKILGTFKSVAVKAPGGGDRRKAQLADIAILTGGQVIS 300

Query: 242 GKPLLIIAEDVEGEALSTLVVNKIRGTFKSVAVKAPGFGDRRKAQLADIAILTGGEVISE 301 GKPLLIIAEDVEGEALSTLVVNKIRGTFKSVAVKAPGFGDRRKA L D+AILTGG+VISE

Sbjct: 241 GKPLLIIAEDVEGEALSTLVVNKIRGTFKSVAVKAPGFGDRRKAMLQDMAILTGGQVISE 300

Query: 301 EEVGLSLETAGIELLGQARKVVVTKDETTIVEGAGDAEAIKGRVAQIRTEIENSDSDYDR 360 EEVGLSLETAGIELLGQARKVVVTKDETTIVEGAGDAEAI GRV+QIR EIENSDSDYDR

Sbjct: 301 EEVGLSLETAGIELLGQARKVVVTKDETTIVEGAGDAEAIAGRVSQIRAEIENSDSDYDR 360

Query: 302 EVGLSLETAGIELLGQARKVVVTKDETTIVEGAGDAEAIKGRVAQIRTEIENSDSDYDRE 361 EVGLSLE+A I LLG+ARKVVVTKDETTIVEGAGD++AI GRVAQIRTEIENSDSDYDRE

Sbjct: 301 EVGLSLESADISLLGKARKVVVTKDETTIVEGAGDSDAIAGRVAQIRTEIENSDSDYDRE 360

Query: 361 EKLQERLXXXXXXXXXXXXXXXTEVELKERKHRIEDAVRNXXXXXXXXXXXXXXXXXXXX 420 EKLQERLTEVELKERKHRIEDAVRN

Sbjct: 361 EKLQERLAKLAGGVAVIKAGAATEVELKERKHRIEDAVRNAKAAVEEGIVAGGGVAFLQS 420

Query: 362 KLQERLXXXXXXXXXXXXXXXTEVELKERKHRIEDAVRNXXXXXXXXXXXXXXXXXXXXX 421 KLQERLTEVELKERKHRIEDAVRN

Sbjct: 361 KLQERLAKLAGGVAVIKAGAATEVELKERKHRIEDAVRNAKAAVEEGIVAGGGVALLHAI 420

Query: 421 XXXXDELKLTGDEATGANIVRVALSAPLKQIAFNAGLEPGVVAEKVSNLEAGHGLNADSG 480 D+ KL GDEATGANIVRVALSAPLKQIAFNAGLEPGV+AEKVSNL AG GLNA +

Sbjct: 421 VPALDDFKLEGDEATGANIVRVALSAPLKOIAFNAGLEPGVLAEKVSNLPAGQGLNAQTN 480

Query: 422 XXXDELKLTGDEATGANIVRVALSAPLKQIAFNAGLEPGVVAEKVSNLEAGHGLNADSGE 48 DELKL G+EATGANIVRVAL APLKQIAFN GLEPGVVAEKV N AG GLNA +GE

Sbjct: 421 PALDELKLEGEEATGANIVRVALEAPLKQIAFNGGLEPGVVAEKVRNSPAGTGLNAATGE 480

Query: 481 EYEDLLAAGVADPVKVTRSALQNAASIAALFLTTEAVVADKPEKA-AAPAGDPTGGMGGM 539 E EDLLAAGVADPVKVTRSALQNAASIAALFLTTEAVVADKPEKA AAPA MGGM

Sbjct: 481 EDEDLLAAGVADPVKVTRSALQNAASIAALFLTTEAVVADKPEKASAAPATGHRFKMGGM 540 Query: 482 YEDLLAAGVADPVKVTRSALQNAASIAALFLTTEAVVADKPEKAAAPAGDPTGGMGGMDF 541 YEDLL AG+ADPVKVTRSALQNAASIA LFLTTEAVVADKPEKAAAPAGDPTGGMGGMDF

Sbjct: 481 YEDLLKAGIADPVKVTRSALQNAASIAGLFLTTEAVVADKPEKAAAPAGDPTGGMGGMDF 540

Query: 540 DF 541 DF

Sbjct: 541 DF 542 
Table 1/5: Transkriptase alpha chain of $N$. farcinica RPOA_NOCFA (Q5Z1K9)

\begin{tabular}{|c|c|c|}
\hline Description & SwissProt Accession \# & Organism \\
\hline _RHOSR & Q0S3E7 & Rhodococcus sp. strain RHA1 \\
\hline _MYCSS & Q1BD08 & Mycobacterium sp. strain MCS \\
\hline _9MYCO & Q1T8C7 & Mycobacterium sp. KMS \\
\hline _9MYCO & Q1TR19 & Mycobacterium sp. JLS \\
\hline _MYCFV & Q27E10 & Mycobacterium flavescens $P Y R-G C K$ \\
\hline -MYCVN & Q263M4 & Mycobacterium vanbaalenii PYR-1 \\
\hline RPOA_MYCTU & P66701 & Mycobacterium tuberculosis \\
\hline RPOA_MYCBO & P66702 & Mycobacterium bovis \\
\hline RPOA_MYCPA & Q73S43 & Mycobacterium paratuberculosis \\
\hline RPOA_MYCLE & Q9X798 & Mycobacterium leprae \\
\hline
\end{tabular}

Table 1/6: Enolase of $N$. farcinica ENO_NOCFA (Q5YQ30)

\begin{tabular}{|l|l|l|}
\hline Description & \multicolumn{1}{|c|}{ SwissProt Accession \# } & Organism \\
\hline RHOSR & Q0S4I1 & Rhodococcus sp. strain RHA1 \\
\hline _MYCSS & Q1B439 & Mycobacterium sp. strain MCS \\
\hline 9MYCO & Q1TAN9 & Mycobacterium sp. KMS \\
\hline _9MYCO & Q1TX18 & Mycobacterium sp. JLS \\
\hline _MYCFV & Q275H2 & Mycobacterium flavescens PYR-GCK \\
\hline ENO_MYCPA & Q741U7 & Mycobacterium vanbaalenii PYR-1 \\
\hline _MYCVN & Q267Y1 & Mycobacterium tuberculosis \\
\hline ENO_MYCBO & Q7U0U6 & Mycobacterium bovis \\
\hline ENO_MYCTU & P96377 & Mycobacterium tuberculosis \\
\hline ENO_MYCLE & Q9CD42 & Mycobacterium leprae \\
\hline
\end{tabular}

\section{REFERENCES}

Bauer AW., Kirby WM., Sherris JC. and Turck M. (1966). Antibiotic susceptibility testing by a standardized single disk method. Am $J$. Clin Pathol 45: 493-496.

Brubacher JL., Dewitte-Orr SJ., Zorzitto JR., Playle RC., Bols NC. (2003). Redox-active metals in commercial preparations of lipopolysaccharide: implications for studies of cellular responses to bacterial products. Cell Microbiol. 5(4): 233-243

Gang Wu., Lei Nie and Weiwen Z. (2005). Predicted highly expressed genes in Nocardia farcinica and the implication for its primary metabolism and nocardial virulence. Antonie van Leeuwenhock 89(1): 135-146.

Garcia BV., Garcia H. L., Archer DC., and OrozcoTR. (2001). Acute primary superficial nocardiosis due to Nocardia brasiliensis: a case report in an immunocompromised patient. Eur J Epidemiol. 17(11): 1019-1022.

Goodfellow M. (1992). The family Nocardiaceae. The prokaryotes; Vol 2. 2nd Ed., Eds. Balows, A., Truper, H. G. et al., SpringerVerlag New York, 1188-213.

Hamid ME., Maldonado L., Sharaf Eldin GS., Mohammed MF., Saeed NS., Goodfellow M.
(2001) Nocardia africana sp. nov, a new pathogen isolated from patients with pulmonary infections. Clin. Microbiol. 39(2) :

625-30.

http://www.cdc.gov/ncidod/dbmd/diseaseinf o/nocardiosis_t.htm

Isik KJ., Chun YC. and Goodfellow M. (1999). Nocardia salmonicida nom. rev., a fish pathogen. Int. J. Syst. Bacteriol. 49:833-837.

Ishikawa Jun., Atsushi Y., Yuzuru M., Yasutaka H., Haruyo K., Kunimoto H., Tadayoshi S. and Masahiara H. (2004)The complete genomic sequence of Nocardia farcinica IFM 10152. PNAS 101(41):14925-14930.

Koltzscher M., Claudia N., Simone K. and Volker G. (2003). Ca2+-dependent binding and activation of dormant ezrin by dimeric S100P. Mol Biol of the Cell 14(6): 23722384.

Mattow J., Jungblut PR., Schaible UE., Mollenkopf HJ., Lamer S., Zimny-Arndt U., Hagens K., Müller EC., Kaufmann, SH. (2001). Identification of proteins from Mycobacterium tuberculosis missing in attenuated Mycobacterium bovis BCG strains. Electrophoresis 22(14):2936-46. 
Minnikin DE, Alshamaony L. and M. Goodfellow. 1975. Differentiation of Mycobacterium, Nocardia and related taxa by thin-layer chromatographic analyses of whole-cell methanolysates. J. Gen. Microbiol. 88:200-204.

Mogahid EE, Kanury VS., Zaved S., Dinesh SK., Rashmi T., Nageeb SS., Moawia MM. and Mohamed H. (2007) Proteomics of Nocardia africana (SD769) recently isolated from patients with pulmonary infection in Sudan. Biomacromol Mass Spectrom 1(3): 171-177.

Orchard, V. A. (1981). The ecology of Nocardia and related taxa. Zentralbl. Bakteriol. Suppl. 11: $167-80$.
Peter R., Jungblut ECM., Jens M. and Stefan HEK. (2003) Proteomics Reveals Open Reading Frames in Mycobacterium tuberculosis H37Rv Not Predicted by

Roberts GD., Koneman EW. and Kim YK. (1991). Mycobacterium, p.304-339. In A. Balows, W. J. Hausler, Jr., K. L. Herrmann, H. D. Isenberg, and H. J. Shadomy (ed.), Manual of clinical microbiology, 5th ed. American Society for Microbiology, Washington, D.C.

Weichart D., Querfurth N., Dreger M., HenggeAronis R. (2003). Global role for ClpPcontaining proteases in stationary-phase adaptation of Escherichia coli. J. Bacteriol. 185(1): 115-125.

\title{
ARABIC SUMMARY
}

\author{
إلتهاب الصدر المسبب بالنوكارديا و مشابهته لمرض الدرن (دراسة باكتيرية و بروتيومية)

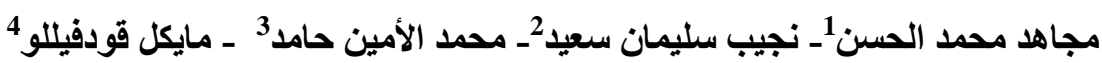

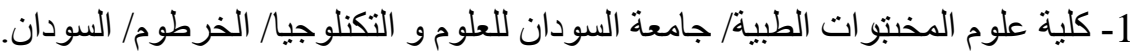

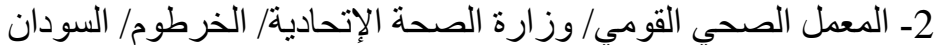

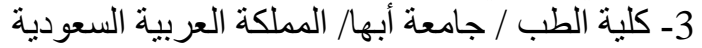 \\ 4- مدرسة الاحياء/جامعة نيو كاسل/ المملكة المتحدة المداءة \\ هدفت هذه الدر اسة لمعرفة م عدل حدوث الاصـابة بالنوكارديا عند المرضى السودانيين المشتبه في اصابتهم بالسل الرئوي ,

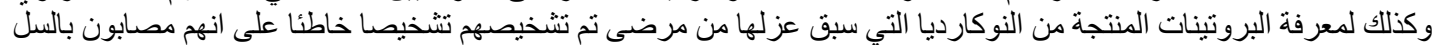

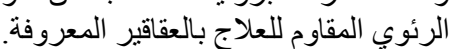

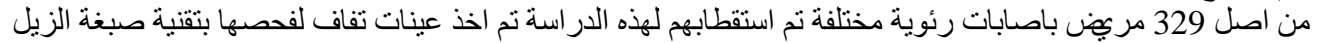

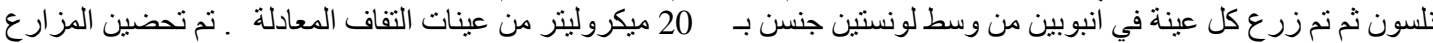

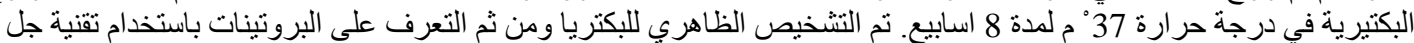

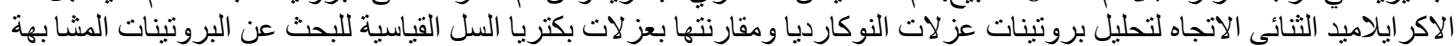

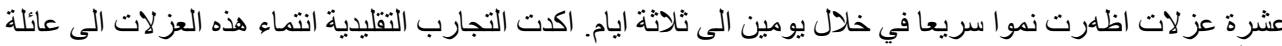 \\ في قاعدة البيانات العالمية.

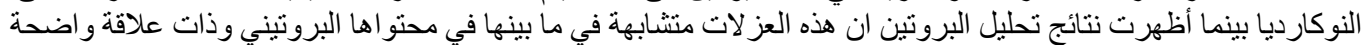 \\ بيروتينات بكتيريا السل.

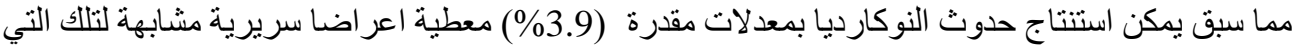

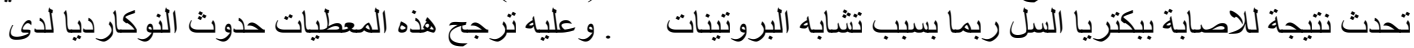

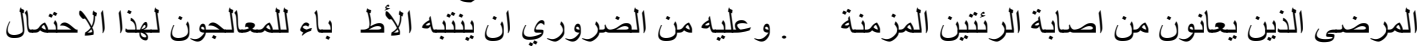

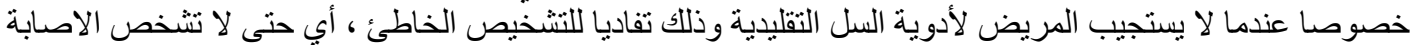 \\ بالنوكارديا الرئوية كاصابة ببكتريا السل المقاومة للعقاقير.
}

